Journal of Social Sciences 6 (1): 50-54, 2010

ISSN 1549-3652

(C) 2010 Science Publications

\title{
Effects of Perceived Fitness Level of Exercise Partner on Intensity of Exertion
}

\author{
Thomas G. Plante, Meghan Madden, Sonia Mann, Grace Lee, \\ Allison Hardesty, Nick Gable, Allison Terry and Greg Kaplow \\ Department of Psychology, Alumni Science Hall, Room 203, \\ Santa Clara University, Santa Clara, CA 95053-0333
}

\begin{abstract}
Problem statement: Social comparison theory was used to examine if exercising with a research confederate posing as either high fit or low fit would increase the exertion in exercising. Approach: 91 college students were randomly assigned to one of three conditions: Biking alone, biking with a high fit confederate, or biking with a low fit confederate. All participants were instructed to complete $20 \mathrm{~min}$ of exercise at $60-70 \%$ of their maximum target heart rate. Results: Results indicated that participants in the high fit condition exercised harder than those in the low fit condition. However, no mood differences emerged between conditions. Conclusion: Social comparison theory predicts exercise outcome such that participants gravitate towards the behavior (high fit or low fit) of those around them.
\end{abstract}

Key words: Exercise, social comparison, perceived fitness, mood, exertion

\section{INTRODUCTION}

Research has demonstrated many physical and psychological benefits of exercise including reduced risks of cardiovascular disease, hypertension, diabetes, cancer and obesity (Center for Disease Control and Prevention, 2006; Blair et al., 1989; Brukner and Brown, 2005; Byers et al., 2002; Pate et al., 1995; Bryan et al., 2007; Morgan, 1985) as well as psychological disturbances such as depression, anxiety and stress disorders (Plante and Rodin, 1990; Plante, 1999; Kennedy and Newton, 1997). Researchers generally agree that exercise provides many benefits for both physical and mental health (Center for Disease Control and Prevention, 2006; Blair et al., 1989; Brukner and Brown, 2005; Byers et al., 2002; Pate et al., 1995; Bryan et al., 2007; Morgan, 1985; Plante and Rodin, 1990; Plante, 1999; Kennedy and Newton, 1997). Although research on the many benefits of exercise is abundant, there is surprisingly little research on the psychological and behavioral effects of exercising with others. The limited available research examining social exercise has demonstrated health and mood advantages and disadvantages of exercising with a partner (Plante et al., 2001; 2003).

Social comparison theory may offer a helpful framework for understanding the effects of exercising with others. The theory states that "humans have a drive to assess how they are doing and in order to assess how they are doing, they seek standards against which to compare themselves. When objective standards are not available, people look to their social environments and engage in comparison with available others" (Corning et al., 2006). Social comparison theory has been applied to various research areas that may explain why people are motivated to engage in healthpromoting or health damaging behaviors (Festinger, 1954).

For example, in an investigation on how social influences encourage healthy behaviors such as exercise, perceived behaviors of peers influenced the behavior of others such that individuals were likely to mimic the behavior of those around them (Festinger, 1954; Luszczyska et al., 2004). Individuals feel the need to engage in socially acceptable behaviors, such as exercising, when observing others doing the same (Luszczyska et al., 2004).

Additional research demonstrates that mood and energy levels are altered when exercising in the presence of others or in front of a mirror. For example, women who exercised with a partner or in front of a mirror experienced an increased level of exhaustion and decreases in feelings of revitalization while exercising compared to women who exercised either alone or without mirrors (Ginis et al., 2006). Although some people may feel self-conscious exercising in the

Corresponding Author: Thomas G. Plante, Department of Psychology, Alumni Science Hall, Room 203, Santa Clara University, Santa Clara, CA 95053-0333 
presence of others, research demonstrates that individuals often engage in exercise for social interaction. The likelihood of joining and staying motivated during exercise increases when friends or peers engage in those same activities (Faulkner et al., 2008; Laverie, 1998).

In the present study, social comparison theory was used to determine if exercising with someone perceived to be either high or low in fitness would alter the exercise experience and behavior of research participants. In particular, we examined mood and the level of exertion in subjects when exercising with someone who the participant believed to possess either a high or low level of fitness. We hypothesized that the level of effort exerted by participants would match the perceived fitness of their partner such that research participants would mimic the exercise behavior of those around them during exercise.

Exercise in this study was defined as biking either alone or with a partner in one of three experimental conditions. In two experimental conditions, the participant exercised with a research confederate as their partner. One of these conditions used a "high fit" confederate, while the other condition used a "low fit" confederate. In a third and control condition, the participant exercised on a stationary bicycle alone. All of the participants in the experiment completed the same intensity and length of time of physical activity required to meet the daily recommended criteria suggested by the Center for Disease Control and Prevention (2009).

\section{MATERIALS AND METHODS}

Participants: The sample consisted of 91 undergraduate students at a West coast private Catholic university (43 females, 48 males, $M=18.84$ years, SD = 1.07). All subjects were enrolled in a general psychology course and received research participation credit. The project received approval from the human subjects committee at the university where it was conducted.

\section{Measures:}

Activation-Deactivation Adjective Check List (ADACL) (Thayer, 1978; Thayer, 1986): The AD-ACL is a brief, frequently used self-report checklist designed to measure momentary mood states associated with exercise with reported adequate reliability and validity used in a number of investigations involving exercise.
Perceived Exertion Scale (Borg scale, PES) (Borg, 1982): The PES was used to evaluate the participants' perceived level of exertion where $6=$ very light exertion and $20=$ very hard exertion. The PES is often used in exercise research and has adequate reliability and validity.

Paces Activity Enjoyment Scale (PACES) (Kendzierski and DeCarlo, 1991): The PACES scale includes18 bipolar items on which individuals rate themselves on a 7 point Likert scale. The scale measures the amount of enjoyment individuals perceive themselves to have experienced during an exercise activity. Sample scale items include "I find it energizing/I find it tiring "and" I enjoy it/I hate it." The authors report that PACES has excellent internal consistency, stability and validity.

Several researchers developed Likert scales: Several 10 point Likert scales developed by the authors measuring each participant's current level of perceived stress, how participants felt while exercising next to someone who was either posing as high fit or low fit. The value of 1 indicated high stress, low enjoyment, low level of comfort and feeling low fit while the value of 10 indicated very relaxed, very enjoyable, very comfortable and feeling very fit.

Procedure: Participants enrolled in the study to complete a requirement for a general psychology undergraduate class. On the day prior to their scheduled laboratory session, participants received an email to remind them of the experiment and confirmed their appointment. The participants were told to wear comfortable and exercise appropriate clothing.

Prior to beginning of the experiment, participants reviewed and signed consent forms agreeing to participate. Then, they were administered the preexercise questionnaires and their height and weight was recorded. The lab assistant then placed heart rate monitors on the participants' upper torsos and gave them a wristwatch that displayed their heart rates. Participants were randomly assigned to one of three experimental conditions. The length and intensity of exercise were the same and included biking for $20 \mathrm{~min}$ on a stationary bike. Participants were instructed to keep their heart rates at a moderate level of $60-70 \%$ maximum heart rate (i.e., about $130 \mathrm{bpm}$ for college students). The conditions included: biking alone in a control condition, biking with a same gender "high-fit" confederate and biking with a same gender "low-fit" confederate. 
For the purposes of this experiment we operationally defined "high fit" as someone who wore athletic clothing, exercised intensely and stated to the experimenter in the presence of the research subject, "I am so glad you had a fitness study, I love exercising" while mounting the bike. A "low-fit" confederate wore non-athletic gear (e.g., jeans, slippers), barely exerted themselves and stated to the experimenter in the presence of the research subject, "I don't know why I signed up for this experiment, I hate exercising" while mounting the bike.

In all conditions, confederates entered the room after the participant had arrived and asked if they were in the right place for the fitness study. This was done to minimize suspicions that confederates were research assistants.

After exercising, the participant and the confederate were asked to rate their perceived level of exertion according to the PES/Borg Scale (Borg, 1982). After completing the experiment, the experimenter debriefed the participants and informed them about the purpose of the study. The confederates' identity was revealed (i.e., that they were part of the experiment) and participants were asked not to discuss the experiment with others to avoid future possible participants from learning about the purpose of the study. The participants were thanked for their time and provided with course credit.

\section{RESULTS}

A $2 \times 3$ Analysis Of Variance (ANOVA) was used to analyze the data. The only exception was for the measure of mood post-exercise scores where a $2 \times 3$ Analysis Of Covariance (ANCOVA) was used with pre laboratory exercise mood scores used as covariates.

The manipulation check was successful in that participants who exercised with high fit confederates perceived them as high fit while participants who exercised with low fit confederates perceived them as low fit. Participants ranked confederates on a 10-point scale where 10 was defined as being high fit and 1 was defined as being low fit. The average rating for high fit confederate was $7.8(\mathrm{SD}=1.2)$ while the average rating of low fit confederates were 5.2 $(\mathrm{SD}=1.3 ; \mathrm{F}(2,63)=24.04, \mathrm{p}<0.001)$.

Pulse rate and exertion results indicated that participants in the high fit group had higher pulse rates and worked harder than participants in the low fit and control groups $(\mathrm{F}(2,90)=9.05, \mathrm{p}<0.001)$. For example, females who exercised with high fit confederates had an average pulse rate of 133 beats $\min ^{-1}(\mathrm{bpm})(\mathrm{SD}=24.35)$ while females who exercised with low fit confederates had an average pulse rate of $119 \mathrm{bpm}(\mathrm{SD}=13.54)$. Males who exercised with a high fit confederate had an average pulse rate of $124 \mathrm{bpm}(\mathrm{SD}=18.39)$ while males who exercised with a low fit confederate had an average pulse rate of $99 \mathrm{bmp}(\mathrm{SD}=8.46)$. Additionally, females in experimental groups worked harder and had higher pulse rates than males, yielding a significant gender interaction $(F(1,90)=20.38, \mathrm{p}<0.001)$.

Participants in experimental groups felt they exerted themselves more than participants in control groups $(\mathrm{F}(2,91)=3.42, \mathrm{p}<0.05)$ and females ranked their exertion levels higher than males $(\mathrm{F}(1,91)=4.38$, $\mathrm{p}<0.05)$.

A non significant trend demonstrated that participants in the high fit conditions were the least calm while those in the control group were the most calm $(\mathrm{F}(2,91)=2.87, \mathrm{p}=0.063)$. Females in the high fitness condition were the most uncomfortable and the least $\operatorname{calm}(\mathrm{F}(2,91)=3.90, \mathrm{p}<0.05)$.

Interestingly, participants in the control group enjoyed the exercise activity the most $(\mathrm{F}(2,93)=$ $\mathrm{p}<0.05)$ and reported being the most relaxed $(\mathrm{F}(2,93)=$ $\mathrm{p}<0.05)$.

Surprisingly, there were no significant main effects or interactions for the measures of mood, tiredness, tension and energy with all p's $>0.05$.

\section{DISCUSSION}

Social comparison theory has been applied to a variety of research areas such as perceived physical appearance, wealth, success and failure (Buunk and Gibbons, 1997). The goal of this study was to examine how social comparisons might impact an individual's level of exertion and mood during an exercise routine with a perceived high fit or low fit exercise partner. The results were consistent with social comparison theory predictions when applied to exercise outcome such that participants gravitate towards the exercise behaviors of those around them. Even when all participants, regardless of experimental conditions, were instructed to exercise at a moderate level and keep their pulse rates within a particular range, they mimic the exercise behavior of their exercise partner. Additionally, individuals who exercised alone reported feeling calmer and more relaxed in comparison to individuals who exercised with a partner.

Implications of this research might suggest that individuals attempting to exercise more intensely could benefit by exercising next to someone they perceive to be high fit. However, high fit individuals would likely not receive those same benefits when working next to someone lower in fitness. A less fit individual might 
influence a higher fit individual to exert themselves less. Thus, a high fit individual might benefit more from either exercising alone or exercising with another high fit individual. Exercising with someone more fit than oneself could promote a higher intensity workout (Daley and Huffen, 2005). However, exercising alone may prove to be more beneficial than exercising with either a high or low fit individual when trying to secure a relaxing exercise experience.

Results from the current study must be considered cautiously. The sample consisted of a generally homogeneous population of generally high fit and healthy undergraduate students at a private university. Furthermore, the sample size was small $(\mathrm{n}=91)$ and the findings may have occurred due to unknown factors. The lab setting of this experiment may not generalize to the real world such as a fitness club or exercise gym experience. Health clubs often have a variety of fitness levels represented, have music, mirrors and other types of cues that differ from a university laboratory setting. Curiously, no group differences were found while measuring mood. One might expect that mood would be impacted by the experience but results showed that it was not in this study. Finally, in examining our manipulation check regarding the difference in perceived fitness level of the confederate subjects, the findings were modest suggesting that perhaps participants experienced the high fit participant as high fit but the low fit participant as being moderately fit.

\section{CONCLUSION}

Future research should further investigate the effects of social comparison theory on exercise behaviors. It would be useful to use a heterogeneous sample with individuals ranging in fitness levels. It would also be useful to repeat this study in a more real world setting, such as an exercise gym or health club.

\section{REFERENCES}

Blair, S.N., H.W. Kohl, R.S. Paffenbarger, D.G. Clarkand and K.H. Cooper et al., 1989. Physical fitness and all-cause mortality. A prospective study of healthy men and women. J. Am. Med. Assoc., 262: 2394-2401. DOI:10.1001/JAMA.262.17.2395

Borg, G.V., 1982. Perceived exertion scale. Med. Sci. Sports Exerc., 14: 377-381. PMID: 7154893

Brukner, P.D. and W.J. Brown, 2005. Is exercise good for you? Med. J. Aust., 183: 538-541. PMID: 16296971
Bryan, A., K.E. Hutchison, D.R. Seals and D.L. Allen, 2007. A transdisciplinary model integrating genetic, physiological and psychological correlates of voluntary exercise. Health Psychol., 26: 30-39. DOI: $10.1037 / 0278-6133.26 .1 .30$

Buunk, B.P. and F.X. Gibbons, 1997. Health, Coping and Well-Being: Perspectives from Social Comparison Theory. Lawrence Erlbaum Associates, New Jersey. ISBN: 0805818588.

Byers, T., M. Nestle, A. Mctiernan, C. Doyke and A. Currrie-Williams et al., 2002. American cancer society guidelines on nutrition and physical activity for cancer prevention: Reducing the risk of cancer with healthy food choices and physical activity. CA: Cancer J. Clin., 52: 92-119. DOI: 10.3322/CANJCLIN.52.2.92

Center for Disease Control and Prevention 2006. Physical Activity for Everyone: Recommendations: How active do adults need to be to gain some benefit?

http://www.cdc.gov/nccdphp/dnpaphysical/recom mendations/adults.htm

Center for Disease Control and Prevention, 2009. Physical activity for everyone: Recommendations: How active do adults need to be to gain some benefit?

http://www.cdc.gov/nccdphp/dnpaphysical/recom mendations/adults.htm

Corning, A.F., A.J. Krumm, J. Angela and L.A. Smitham, 2006. Differential social comparison processes in women with and without eating disorder symptoms. J. Couns. Psychol., 53: 338-349. DOI:10.1037/0022-0167.53.3.338

Daley, A.J. and C. Huffen, 2005. The effects of low and moderate intensity exercise on subjective experiences in a naturalistic health and fitness club setting. J. Health Psychol., 8: 685-691. DOI: 10.1177/13591053030086003

Faulkner, J., G. Parfitt and R. Eston, 2008. The rating of perceived exertion during competitive running scales with time. Psychophysiology, 45: 977-985. DOI: $10.1111 / \mathrm{J} .1469-8986.2008 .00712 . \mathrm{X}$

Festinger, L., 1954. A theory of social comparison processes. Hum. Relat., 7: 117-140. DOI: 10.1177/001872675400700202

Ginis, K.A., S.M. Burke and L. Gauvin, 2006. Exercising with others exacerbates the negative effects of mirrored environments on sedentary women's feeling states. Psychol. Health, 22: 945-962. DOI: $10.1080 / 14768320601070571$

Kendzierski, D. and K.J. DeCarlo, 1991. Physical activity enjoyment scale: Two validation studies. J. Sport Exerc. Psychol., 13: 60-64. 
Kennedy, M.N. and M. Newton, 1997. Effect of exercise intensity on mood in step aerobics. J. Sports Med. Phys., 37: 200-204. PMID: 9407751

Laverie, D.A., 1998. Motivations for ongoing participation in a fitness activity. Leisure Sci., 20: 277-302. DOI: 10.1080/01490409809512287

Luszczyska, A., F.X. Gibbons, B.F. Piko and M. Tekozel, 2004. Social comparison and perceived peers behaviors as predictors of nutrition and physical activity: A comparison among adolescents in Hungary, Turkey, Poland and USA. Psychol. Health, 19: DOI: 10.1080/0887044042000205844

Morgan, W.P., 1985. Psychogenic factors and exercise metabolism: A review. Med. Sci. Sports Exerc., 17: 309-316. PMID: 3894867

Pate, R.R., M. Pratt, S.N. Blair, W.L. Haskell and C.A. Macera et al. 1995. Physical activity and public health. A recommendation from the centers for disease control and prevention and the American college of sports medicine. J. Am. Med. Assoc., 273: 402-407. DOI: 10.1001/JAMA.273.5.402

Plante, T.G. and J. Rodin, 1990. Physical fitness and enhanced psychological health. Curr. Psychol. Res. Rev., 9: 3-24. DOI: 10.1007/BF02686764
Plante, T.G., 1999. Could the perception of fitness account for many of the mental and physical health benefits of exercise. Adv. Mind-Body Med., 15: 291-301. PMID: 10555401

Plante, T.G., L. Coscarelli and M. Ford, 2001. Does exercising with another enhance the stress-reducing benefits of exercise? Int. J. Stress Manage., 8: 201-213. DOI: 10.1023/A:1011339025532

Plante, T.G., R. Bogdan, Z. Kanani, M. Babula and E. Ferlic et al., 2003. Psychological benefits of exercising with another. J. Hum. Move. Stud. 44: 93-106. DOI: 10.1037/1072-5245.14.1.88

Thayer, R.E., 1978. Factor analytic and reliability studies on the activation deactivation adjective check list. Psychol. Rep., 42: 747-756. PMID: 674499

Thayer, R.E., 1986. Activation-deactivation adjective check list: Current overview and structural analysis. Psychol. Rep., 58: 607-614. 